psychologists with a comprehensive review of the foundational skills of effective parenting within the context of the contemporary positive psychology movement.

\author{
Dr Michelle Andrews \\ The Educational Psychology Centre, Melbourne, Australia \\ Disclaimer: The author has previously worked at the University of Melbourne with \\ Erica Frydenberg.
}

\title{
References
}

Sawyer Cohen, J., \& Semple, R. (2010). Mindful parenting: A call for research. Journal of Child and Family Studies, 19, 145-151.

Wyatt Kaminski, J., Valle, L., Filene, J., \& Boyle, C. (2008). A meta-analytic review of components associated with parent training program effectiveness. Journal of Abnormal Child Psychology, 36, 567-589.

\section{Therapeutic Metaphors for Children and the Child Within (Second Edition)}

Joyce C. Mills and Richard J. Crowley

Routledge New York, 2014, 215 pp., \$52.68 (AU paperback) IBSN: 978-0-415-70810-4.

doi:10.1017/edp.2015.3

Therapeutic Metaphors for Children and the Child Within is best for those working with children and adolescents who are interested in counselling, play therapy and seeking alternatives in counselling settings.

Mills and Crowley draw upon the teachings from the late psychiatrist Dr Milton $\mathrm{H}$. Erikson (1901-1980), who specialised in hypnosis, family therapy, solution-focused brief therapy and neuro-linguistic programming.

The main concept of this book is the use of symbolic language of metaphor and storytelling in counselling. It is well regarded in both eastern and western cultures and documented by Kopp (1971) that the 'use of metaphor is a primary vehicle for teaching' (p. 6).

The book is broken into four main parts. Parts I and II (Chapters 1-6) walk through the elements of metaphor and how to create stories with the client. It is apparent that Parts I and II are practically the same book that was released originally in 1986, as the case studies presented have dated pop culture references (e.g., of the recent release of E.T. the Extra Terrestrial).

A lot of research has taken place in the past 30 years; it would have been in the author's best interest to include and acknowledge some of the advances of science (especially in neuroscience) and other counselling techniques and interventions that can also help explain and illustrate the functional use of metaphors (and vice versa). This could have been achieved, as some of the case studies presented used metaphor in such a way that parallels could be drawn to address CBT concepts in creative and illustrative ways and not just constrained to the methods we are familiar with from typical psychological training.

Part III (Chapters 7-8) of this text is where the applications of metaphor are demonstrated in a much more succinct manner and illustrate the potential effectiveness for 
children and adolescents. In these chapters, metaphor extends out into other elements of creativity, including art, board-games and favoured cartoon characters, to help the client draw (metaphorically and literally) solutions and consider resources to their assigned concerns. This is a highlight of the text that may inspire the practitioner to utilise other methods in counselling.

Part IV (Chapters 9-10) demonstrates how elements of storytelling (StoryPlay therapy) and art therapy have been used in more recent times in response to traumatic events, including the Hurricane Iniki disaster in 1992 and September 11, 2001. It would have been ideal to see more of this area explored and discussed, especially in the area of trauma research in a post-September 11 world.

This book will be of interest to some Educational and Developmental psychologists, especially those working with children and adolescents, who are seeking alternative and creative methods, as there is no 'one size fits all' approach to counselling. It is worth visiting this text and 'allow ourselves to "enter the child within"' (p. 20) as a means to help children and adolescents.

Kathryn Kallady

Educational and Developmental Psychologist, Melbourne, Australia

\section{Reference}

Kopp, S. (1971). Guru: Metaphors from a psychotherapist. Palo Alto, CA: Science \& Behaviors Books.

\section{Early Childhood Theories and Contemporary Issues An Introduction}

Edited by Mine Conkbayir and Christine Pascal

Bloomsbury Academic, 2014, 211 pp., \$34.99 (AU paperback), ISBN: HB978-1-7809-3656-7.

doi:10.1017/edp.2015.4

This book presents a summary of a selection of theories related to early child development (0-3 years old approximately), discusses each theory and then outlines the relation of the theory to practice. It is set out in chronological order, beginning with Rousseau (1712-1778), and moves through the contribution of some well-known theorists, to consider the findings of neuroscience in the early years. The final section addresses the possibility of reconceptualising early years education in the light of the evolution of the theories, as well as how to undertake action research in such settings.

By bringing to the reader an understanding of the theories underpinning psychological practice in this field, it aims to ensure high quality, informed responses to the needs of children, their families and communities.

Each chapter follows a similar pattern, with a summary of the crucial aspects of the theory, discussion about it, practical tips on how to recognise the theory in action, and ways to test these ideas out within the child's setting. This is followed by questions for reflection, which encourage the practitioner to consider the pertinence of the theory or concept to his or her work. Case studies are included throughout, also with questions that further encourage reflective practice. Summaries at the end of each chapter give 